\title{
Green Frame Aggregation Scheme for Wi-Fi Networks
}

\author{
Maha Alaslani, Ahmad Showail, and Basem Shihada \\ Computer, Electrical and Mathematical Sciences and Engineering Division \\ King Abdullah University of Science and Technology (KAUST) \\ Thuwal, Saudi Arabia \\ Email:\{maha.aslani, ahmad.showail, basem.shihada\}@kaust.edu.sa
}

\begin{abstract}
Frame aggregation is a major enhancement in the IEEE 802.11 family to boost the network performance. The increasing awareness about energy efficiency motivates the rethink of frame aggregation design. In this paper, we propose a novel Green Frame Aggregation (GFA) scheduling scheme that optimizes the aggregate size based on channel quality in order to minimize the consumed energy. GFA selects an optimal sub-frame size that satisfies the loss constraint for realtime applications as well as the energy budget of the ideal channel. This scheme is implemented and evaluated using a testbed deployment. The experimental analysis shows that GFA outperforms the conventional frame aggregation methodology in terms of energy efficiency by about $6 \times$ in the presence of severe interference conditions. Moreover, GFA outperforms the static frame sizing method in terms of network goodput while maintaining the same end-to-end latency.
\end{abstract}

Index Terms-Frame aggregation, IEEE 802.11n, A-MPDU, Energy consumption, Frame error rate.

\section{INTRODUCTION}

The last decade has seen a dramatic global growth in wireless communication systems. Statistics show that the number of wireless subscriptions in a any given country have exceeded its own population. As the usage of wireless networks increases, energy efficiency of mobile devices has become a real challenge. Currently, many researchers are focusing on energy consumption of these devices across various layers of the communication system.

The IEEE 802.11 group have introduced several improvements to both physical and MAC layers in IEEE 802.11n/ac in order for it to become the baseline technology in next generation WLANs. In order to achieve high throughput and efficiency, IEEE 802.11n introduces two frame aggregation techniques, namely MAC Service Data Unit Aggregation (A-MSDU) and MAC Protocol Data Unit Aggregation (AMPDU). These frame aggregation methods can increase the throughput under ideal channel conditions by reducing both the headers and timing overheads [1]. However, a large aggregated frame causes other stations in the network to wait longer before they get a transmit opportunity. Moreover, under errorprone channels, a single bit error in a large aggregated frame may waste a long period of channel time and lead to lower network performance and energy efficiency.

Several recent research papers in the field of wireless networking have studied IEEE 802.11 MAC frame aggregation.
Most of the existing work focused on increasing throughput and minimizing delay. For example, Kim et al. [2] analyzed the effect of IEEE 802.11n A-MPDU and A-MSDU frame aggregation on the throughput of the network. A model based on an enhanced discrete time markov chain is proposed to study frame aggregation post-backoff behavior. Lee et al. [3] explored a multiple-receiver frame aggregation scheme to boost the capacity of the video traffic in WLANs. They found that the number of video streams that can be supported on these kind of networks depends heavily on how frame aggregation is implemented. Their multiple-receiver scheme increases the number of supported video streams by a factor of 2. Another simple frame aggregation scheduler is presented by Selvam et al. [4]. It selects the frame size and aggregation type based on the duration of frame transmit opportunity. This method outperformed the state of the art fixed size A-MPDU methods in lightly loaded networks. Zhihui et al. [5] proposed A-MSDU frame size adaptation algorithm under error-prone channels for both uni-directional and bi-directional transfers. Their analytical model is built based on Bianchis model. In a similar work, Teymoori et al. [6] proposed a method to obtain the optimal frame size based on a constrained convex optimization problem. On the other hand, Error-Sensitive Adaptive Frame Aggregation algorithm (ESAFA), proposed by Moh et al. [7], adjusted the aggregate size dynamically according to the application acceptable frame error rate.

There is very limited work on energy efficiency for frame aggregation in WLANs. Keranidis et al. [8] investigated the IEEE 802.11 MAC-layer enhancements and its impact on energy consumption in wireless devices. They showed that frame aggregation mechanisms can reduce the energy consumption by $75 \%$. In a similar effort, Seungwoo et al. [9] introduced a frame aggregation scheme that enhances energy efficiency by adjusting the number of sub-frames per aggregate according to the current battery capacity. However, this scheme may not be useful in the case of A-MPDU frame aggregation where only corrupted sub-frames are being retransmitted. Moreover, this scheme transmits a single frame only i.e., disabling frame aggregation, when the channel quality exceeds a specific threshold which may result in severe drop in the throughput. Finally, Zhihui et al. [10] proposed Energy Efficiency Frame Aggregation scheduling algorithm (EEFA). EEFA varies the frame size based on bit error rate. It reduces the energy con- 


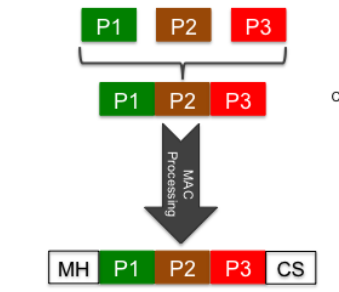

Aggregated MAC Service Data Unit (A-MSDU)

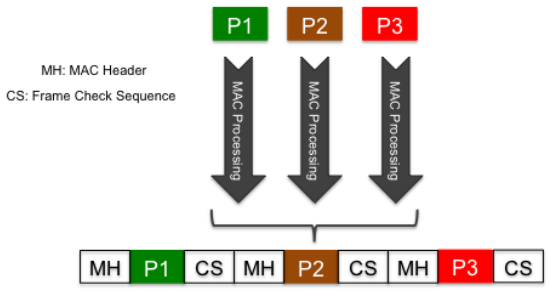

Aggregated MAC Protocol Data Unit (A-MPDU)

Fig. 1: Illustration of A-MSDU and A-MPDU frame aggregation techniques in IEEE 802.11n/ac networks.

sumption by ensuring the data transmission and retransmission are completed at the channel access time. In fact, EEFA divides the transmission time into two phases, one for the aggregated frame and the other is dedicated only for retransmissions. Clearly, this is going to impose a huge overhead when the channel quality is high. Furthermore, EEFA estimates the appropriate frame size using a predefined model. Hence, it lacks the online functionality that our proposed scheme is providing as we are going to show later.

In this paper, we are proposing Green Frame Aggregation (GFA), a novel energy efficient frame aggregation scheme for IEEE $802.11 \mathrm{n} / \mathrm{ac}$ wireless devices. GFA estimates the optimal A-MPDU sub-frame size while taking into consideration the energy constraints of error-prone channels. The main objective of GFA is to minimize energy consumption while maximizing network throughput. To the best of our knowledge, this is the first paper that evaluates a power-aware frame aggregation scheme using real hardware measurements.

Our main contributions are as follows:

1) Design a dynamic A-MPDU frame sizing scheme in the light of Quality of Service (QoS) and energy specifications.

2) Characterize the impact of GFA on our IEEE $802.11 \mathrm{n}$ based testbed and show that it achieves high energy efficiency while boosting the network goodput and maintaining the same latency.

The reminder of this paper is organized as follow: The motivation of our solution and the design of our proposed scheme are illustrated in section II. Then the implementation details are described in section III. In section IV, the experimental performance analysis of GFA scheme is presented. Finally, the work is concluded in section V.

\section{Green Frame Aggregation}

In this section, we start by motivating the need for an energy efficient frame aggregation scheme. After that, the design of GFA is illustrated in details. Finally, we describe how does GFA operate.

\section{A. Motivation}

As mentioned earlier, there are two types of frame aggregation in IEEE 802.11n/ac networks, namely A-MSDU and AMPDU. The former creates the aggregate frame by combining multiple MSDUs that are going to the same destination using

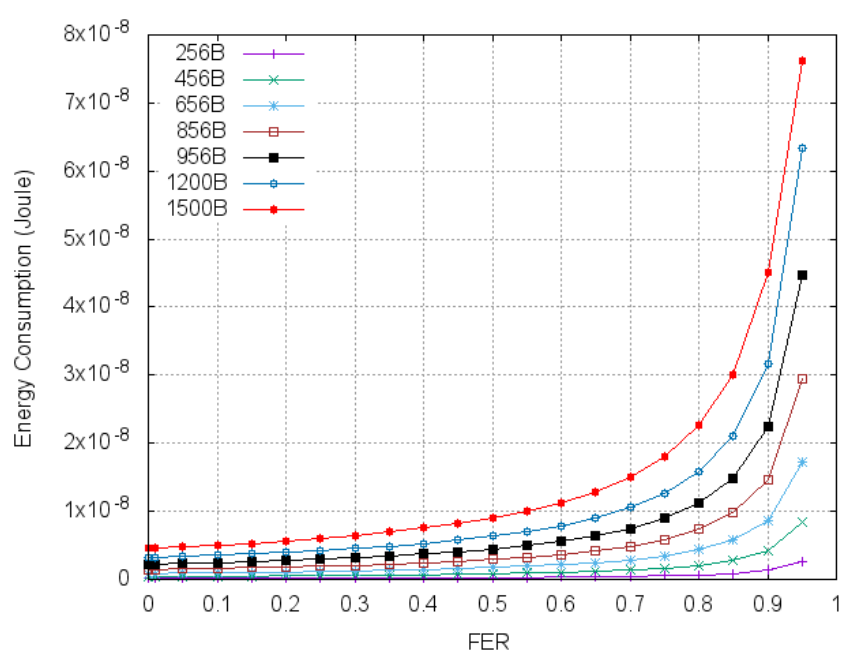

Fig. 2: The effect of varying the A-MPDU sub-frame size on the energy consumption over various channel conditions.

only a single MAC header and a single Frame Check sequence (FCS) trailer. The maximum A-MSDU length can be either 3839 or 7935 bytes based on the advertised High Throughput (HT) capabilities information field. A-MSDU can improve the efficiency especially when there are many small MSDUs. On the other hand, A-MPDU creates much a larger data frame, up to 65535 bytes, by combining multiple MPDUs into a single frame. The actual A-MPDU size may vary according to vendor implementation. In fact, the maximum A-MPDU length may be further limited by the HT receiver capabilities [11].

These two frame aggregation schemes react differently to sub-frame losses. A single bit error in an A-MSDU results in retransmitting the whole A-MSDU again. Alternatively, a lost (or corrupted) sub-frame in any A-MPDU does not disturb the reception of other sub-frames in the same aggregate. This is because each MPDU has its own Frame Check sequence (FCS) to allow the re-transmission of only the corrupted MPDU(s), as illustrated in Fig. 1. Clearly, the aggregation performance is affected by the channel interference level. Noisy channels may cause many bit errors resulting in a lot of aggregate retransmissions, which contribute negatively to the device power consumption. On the other hand, packing frames as aggregates in an error free channel may result in huge power saving since the frame transmission time is going to be shorter and hence the radio active time will also be shorter.

To motivate the problem even further, an experiment is performed to investigate the relationship between the A-MPDU sub-frame size and the energy consumption under error-prone channels. In this experiment, the channel Frame Error Rate (FER) is varied between 0-99\% and the size of the MPDUs i.e., sub-frames, in the aggregated frame is varied within the allowed range (between 256 and 1500 bytes) which is limited by Linux configuration. The implementation setup is described in details in Sec. III. The energy consumption is calculated using the equations in the following subsection. Fig. 2 shows that it is possible to reduce the energy consumption significantly 
by simply varying the A-MPDU sub-frame size based on the channel interference level. To address this issue, a new scheme is developed for estimating the optimal A-MPDU sub-frame size across various channel conditions without sacrificing the network performance. The main goal of the proposed scheme is to increase the energy efficiency of frame aggregation used by IEEE $802.11 \mathrm{n} / \mathrm{ac}$ wireless devices. In the next section, we are going to illustrate how GFA selects the optimal A-MPDU sub-frame size based on the channel quality and the energy budget.

\section{B. Design}

Most of previous efforts in this direction rely on estimating the channel Bit Error Rate (BER) which may not reflect the acceptable Frame Error Rate (FER) of most real time applications. Alternatively, GFA takes into consideration the FER requirements of real-time applications and adjusts the sub-frame size accordingly. Most loss tolerant traffic requires the FER to be within 5\% [12]. Hence, GFA uses this error rate threshold to prevent degrading network performance when trying to maximize energy efficiency. Moreover, this threshold minimizes the overhead of varying the sub-frame size when the FER is within the acceptable limits. It is well known that high throughput can be obtained by transmitting aggregates with the maximum sub-frame size under the best channel condition. Hence, GFA tries to utilize the energy budget associated with the maximum sub-frame size. In this case, our objective function is as follows:

$$
E_{\text {min }}=P \times \frac{L}{R}
$$

where $E_{\min }$ is the minimum energy consumption, $P$ is the power needed to transmit one bit at time, $L$ is the maximum MPDU size and $\mathrm{R}$ is the transmission rate.

In A-MPDU frame aggregation, only corrupted MPDUs need to be retransmitted again. Since the original transmission and the following retransmission are unrelated events, the expected number of transmissions needed to successfully deliver one frame is estimated as

$$
N=\frac{1}{1-F E R}
$$

where $F E R$ is the current Frame Error Rate. So, the energy consumption for reliable transmission is

$$
E_{\text {min }}=P \times \frac{L}{R} \times N
$$

Using (3), the optimal sub-frame size that satisfies our conditions can be calculated from the following relationship

$$
L=\frac{E_{\min } \times R}{P \times N}
$$

According to (4), GFA updates the A-MPDU sub-frame size whenever the Frame Error Rate changes taking into account the FER threshold.

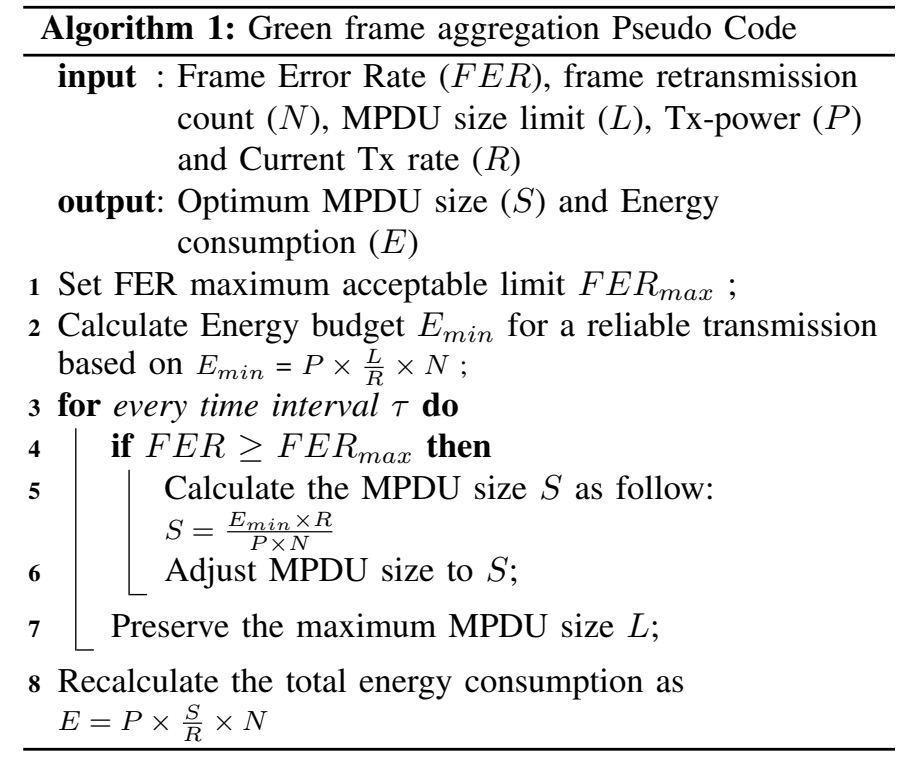

\section{Operation}

In GFA, the decision of frame sizing is mainly based on the channel condition. If the channel quality is less than a predefined threshold, then GFA calculates a new MPDU size to satisfy the requirements; otherwise the maximum MPDU size is used. GFA initially starts with the maximum sub-frame size and calculates the needed energy budget for the reliable data transmission. For each specific time window, GFA senses the channel and estimates the channel FER to determine the suitable MPDU size for the next session. The operation of GFA is illustrated in Algorithm 1. Basically, GFA adds a single additional step on top of the default A-MPDU implementation to select the optimal sub-frame size.

\section{IMPLEMENTATION}

GFA is implemented in Linux and evaluated under errorprone channels using a wireless testbed in our campus. In this section, a detailed description of the used testbed is presented both from hardware and software sides.

\section{A. Hardware Setup}

Our testbed is composed of several nodes that are basically PCs. Each of these boxes is equipped with an Intel E7500 core 2 Due processor, 1 GB of RAM and AR9380 wireless card which is IEEE 802.11n compatible Atheros chipset. This wireless interface provides three RF chains and supports up to three transmit and receive spatial streams with a maximum link rate of $450 \mathrm{Mbps}$.

\section{B. Software Setup}

The testbed nodes run Fedora 20 with Linux kernel 3.14.8 and Linux Ath9k wireless driver, which is a Free and Open Source Software (FOSS) developed by Atheros. This driver implements A-MPDU frame aggregation but not A-MSDU. Hence, we will limit our discussion in this paper to A-MPDU frame aggregation. For fair comparison, we disable the rate 


\begin{tabular}{|l|l|l|l|}
\hline Chipset & \multicolumn{3}{|c|}{ AR9380 } \\
\hline Antennas & $1 \times 1$ & $2 \times 2$ & $3 \times 3$ \\
\hline Mode & \multicolumn{3}{|c|}{ Power Consumption (W) } \\
\hline Sleep & \multicolumn{3}{|c|}{0.12} \\
\hline Idle & 0.49 & 0.56 & 0.69 \\
\hline Receive & 0.62 & 0.74 & 0.85 \\
\hline Transmit & 0.98 & 1.75 & 2.45 \\
\hline
\end{tabular}

TABLE I: AR9380 power consumption across various operation modes [13].

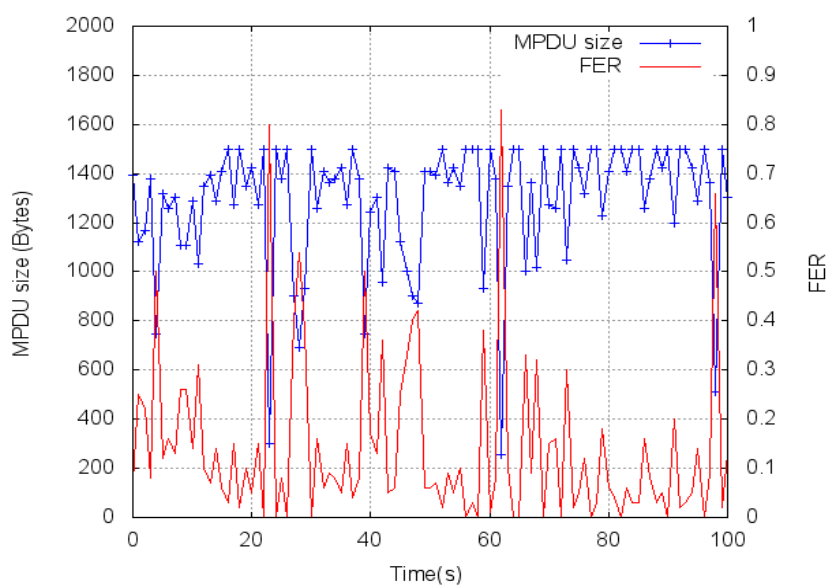

Fig. 3: The dynamic behaviour of GFA over time. The AMPDU sub-frame size is selected based on the channel quality.

control algorithm and fix the link rate to $405 \mathrm{Mbps}$ in all the experiments. Moreover, this high rate allows us to test GFA over high interference levels. To generate traffic in the network we use netperf which is a benchmarking utility that provides performance measurement for various types of flows. We manage to introduce interference in our network by using the crowded $2.4 \mathrm{GHz}$ band which interfere with the production WLAN on our campus.

The maximum and minimum allowed MPDU frame size in our testbed, limited by Linux configuration, are 1500 bytes and 256 byte respectively. Furthermore, the wireless driver limits the A-MPDU aggregated frame length to 32 MPDUs only. On top of that, the actual aggregate size is limited to frame air duration of $4 \mathrm{~ms}$ to comply with the operation regulatory requirements. Moreover, the A-MPDU density, i.e., number of of MPDUs in A-MPDU, is partly dependent on the wireless link rate [14].

The power consumption of the wireless interface cards in our testbed, Atheros AR9380, was measured by Keranidis et al. [13] using an online energy consumption monitoring framework. They varied the operation mode at the transmitter side and repeatedly monitored the energy consumption of each wireless NICs at both the transmitter and the receiver side. After that, specific events were isolated and averaged, such as frame transmission/reception, in order to determine the NIC card power consumption under each specific PHYlayer configuration. The power consumption results using

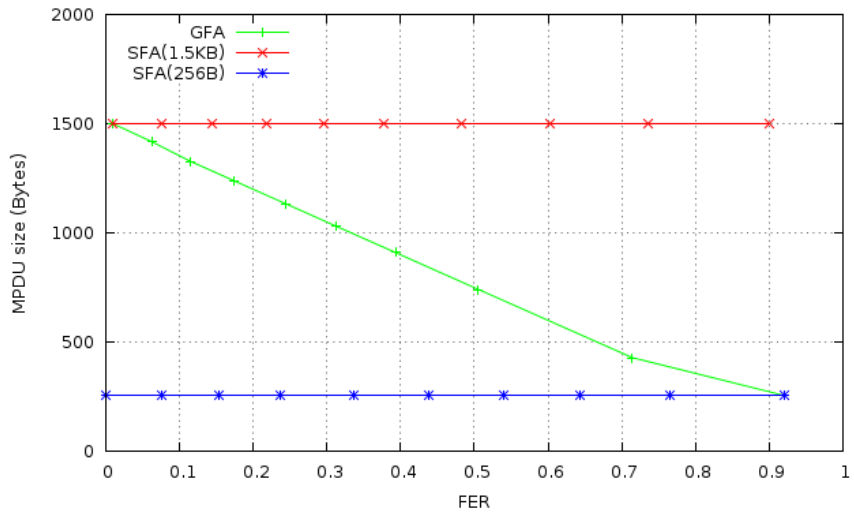

Fig. 4: A-MPDU sub-frame size versus the channel frame error rate for GFA and SFA with the maximum A-MPDU sub-frame size (1500 bytes) and the minimum size (256 bytes).

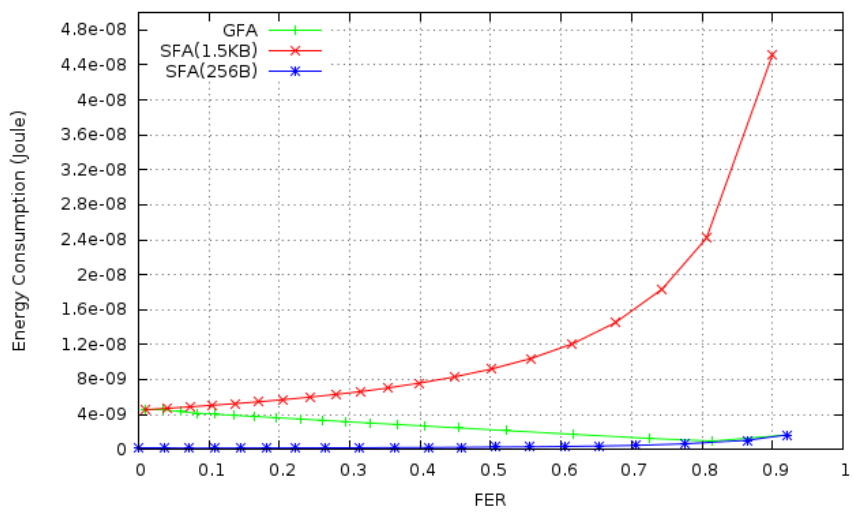

Fig. 5: Energy consumption of both GFA and SFA versus the channel frame error rate.

various operation modes are summarized in Table I. AR9380 consumes around $2.45 \mathrm{~W}$ when the sender is transmitting at 195 Mbps. As shown in the next section, this piece of information will be used to estimate the power needed to send one bit at time.

\section{Performance Evaluation}

In this section, the performance of GFA is evaluated experimentally. First of all, the dynamic nature of GFA is illustrated. Fig. 3 shows that GFA smartly selects the appropriate MPDU size according to channel FER. When the channel quality deteriorates, GFA attempts to operate using smaller MPDUs to save energy.

In order to evaluate the performance of GFA in terms of energy efficiency, several experiments are conducted over various network scenarios. In all the experiments, GFA performance is compared to the Static Frame-size Aggregation (SFA) scheme that is enabled by default in Linux. By default, the A-MPDU sub-frame size is fixed to 1500 bytes which is the maximum supported frame size in our system. To test the other corner case, the same experiments are repeated while fixing the frame size to the minimum supported MPDU size which is 256 bytes. 


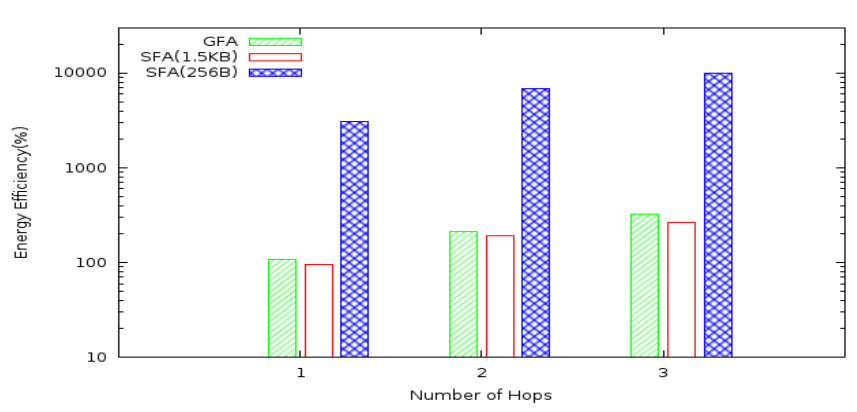

Fig. 6: The energy efficiency under normal channel conditions while varying the hop count. The y-axis shows a logarithmic scale.

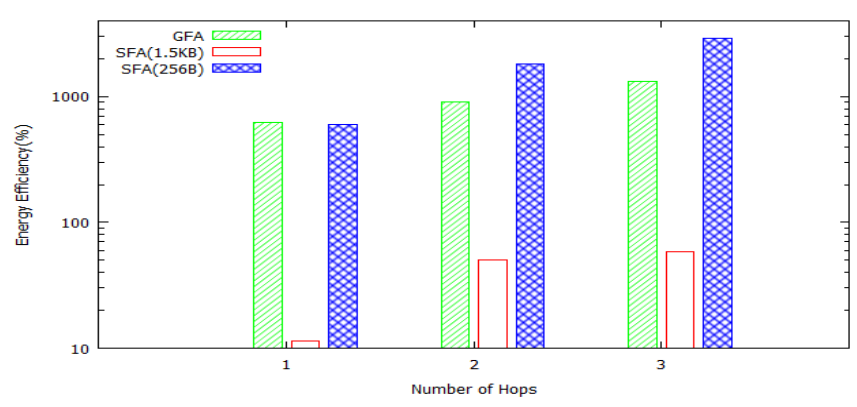

Fig. 7: The energy efficiency under severe interference while varying the hop count. The y-axis shows a logarithmic scale.

It is important to note that the duration of each run is 100 seconds and all our results are averaged over five runs.

First of all, we compare all the schemes in terms of AMPDU sub-frame size and energy utilization while varying the frame error rate. The results for MPDU size as well as energy consumption are shown in Fig. 4 and Fig. 5 respectively. Obviously, Static Frame-size Aggregation (SFA) scheme fixes the A-MPDU sub-frame size regardless of the channel condition. In fact, this comes at the cost of high energy consumption especially when the channel quality is low. On the other hand, GFA adaptively varies the A-MPDU size according to the channel quality. It is clear from these two figures that as the channel quality gets lower, GFA starts outperforming SFA with large sub-frames. In extreme interference conditions, GFA uses the minimum allowed MPDU size which helps saving $6 \times$ more energy. Although SFA with small sub-frame size always maintains low energy consumption, this comes at the cost of low utilization as we are going to show later.

The energy efficiency of the schemes using a multi-hop wireless topology is also analyzed; one to three nodes are added along the path from the sender to the receiver. GFA is implemented in the source as well as all subsequent relay nodes. Energy efficiency can be quantified by the amount of energy drained from the nodes. In fact, it can be calculated by comparing the amount of energy input (the budget) to the amount of energy output (consumption). In this set of experiments, the performance of GFA is also evaluated under a severe interference caused by downloading a very large file

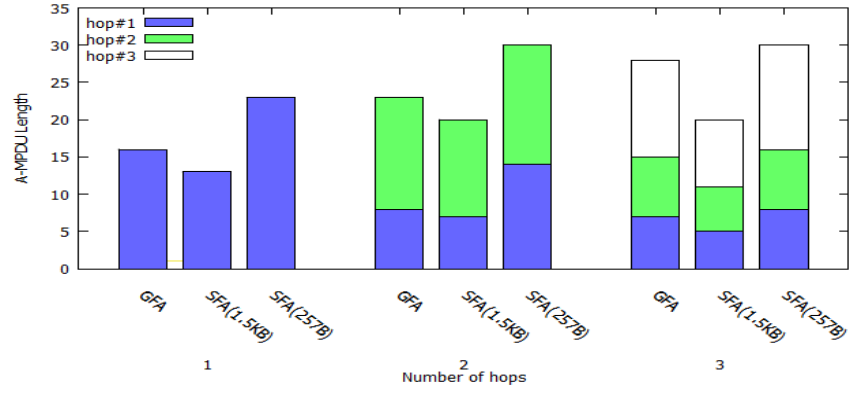

Fig. 8: Average A-MPDU length in the multi-hop network.

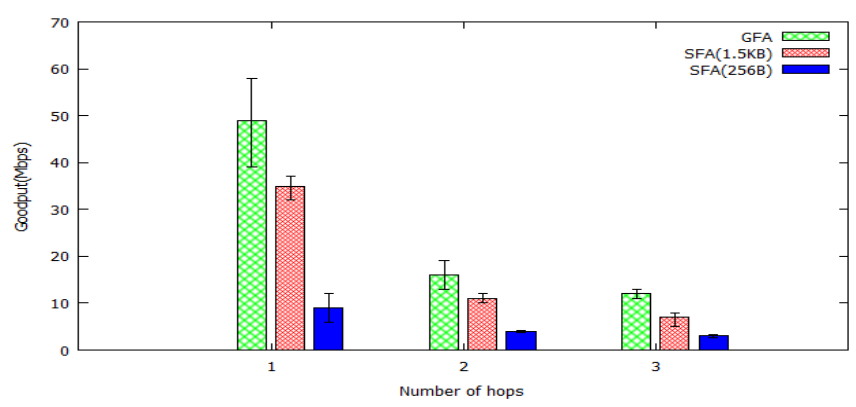

Fig. 9: Average goodput in multi-hop networks.

between two nodes in the network. The aggregated energy efficiency results over normal channel conditions, FER is about $7 \%$, and over the channel with high interference, FER is more than $60 \%$, are shown in Fig. 6 and Fig. 7, respectively. These figures show that GFA is able to boost the energy efficiency across various channel conditions. In the case of high channel quality, the efficiency is increased up by $8 \%, 10 \%$, and $22 \%$ in the 1-hop, 2-hops, and 3-hops scenarios respectively when compared to the SFA with large A-MPDU sub-frames. Although using SFA with small sub-frames increases the energy saving tremendously, this comes at the cost of lowering the throughput by an order of magnitude as shown later. Over noisy channels, GFA improves the energy consumption significantly as well. At extremely low quality channels, Fig. 7 shows that GFA has increased the efficiency by $6 \times$ over the single hop scenario compared to the default static frame size. As the number of hops increases, GFA increases the energy efficiency by $9 \times$ and $13 \times$ for the 2 and 3-hop scenarios respectively.

The average A-MPDU length for various hop counts is shown in Fig. 8. As described earlier, GFA dynamically selects the MPDU size that suits the channel condition observed in a specific time interval. Hence, when the MPDU size gets smaller in response to high interference, the aggregated frame (A-MPDU) gets longer as more sub-frames can fit in the allowed frame air time. This is considered an advantage in low quality channels as only corrupted frames in an A-MPDU gets retransmitted, resulting in increased network goodput. Over a single hop, the average A-MPDU length for GFA is about 16 MPDUs compared to 25 MPDUs for SFA with small subframes and only 12 MPDUs in the case of static large sub- 


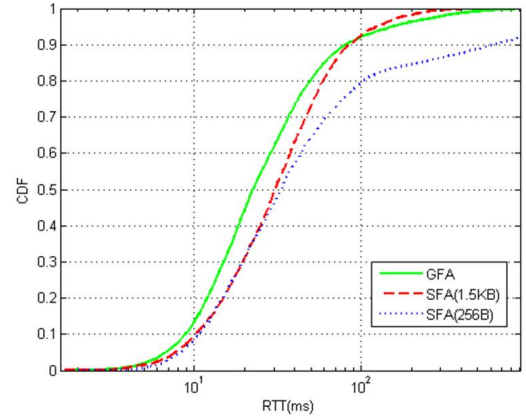

(a) One hop

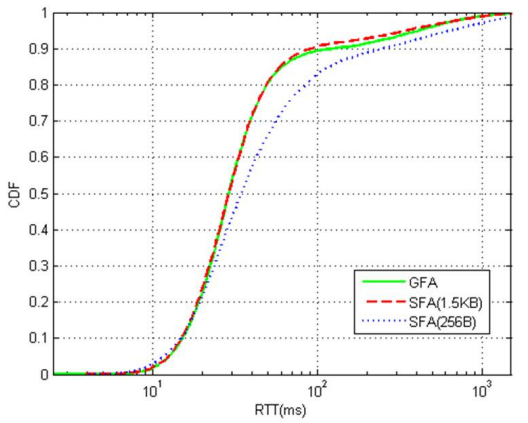

(b) Two hops

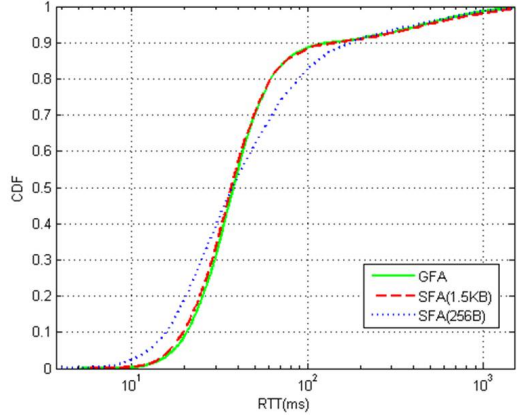

(c) Three hops

Fig. 10: Latency performance of GFA and SFA. The x-axis shows a logarithmic scale.

frames. As expected, the aggregate length per station is going to decrease as the number of hops increases due to more collisions and imperfect spatial reuse. It is worth noting that using static small A-MPDU sub-frame size will increase the total A-MPDU length. However, the overhead associated with these sub-frames will affect the throughput especially when the channel quality is good.

The performance of GFA in terms of network goodput and end-to-end delay is also analyzed over both normal and severe interference conditions. About $70 \%$ of the experimental time is run over normal FER conditions and the remaining 30\% is run over the severe noise level. As shown earlier, GFA increases the overall A-MPDU length which boosts the overall goodput. The average goodput per hop for all schemes is plotted in Fig. 9. The error bars in this figure represent the $95 \%$ confidence intervals. GFA increases the goodput by up to $40 \%$ compared to the SFA with big A-MPDU sub-frame size. On the other hand, GFA increases the total goodput by more than $80 \%$ compared to static small sub-frames. As expected, the overall goodput drops by $1 / 2$ and $1 / 3$ for the 2-hops and 3hops respectively. In addition to the increase in goodput, GFA also achieves almost the same end-to-end delay compared to the static schemes shown in Fig. 10. The reason for this is the fact that transmitting A-MPDUs with smaller sub-frames leads to improved data transmission reliability.

\section{CONCLUSIONS}

In this paper, a novel, power-aware frame aggregation scheme for IEEE $802.11 \mathrm{n} / \mathrm{ac}$ based wireless networks called GFA is proposed. It dynamically chooses the appropriate AMPDU sub-frame size based on channel quality. GFA efficiently utilizes the energy budget that is associated with the maximum A-MPDU sub-frame size. GFA is implemented and evaluated on a Linux based wireless tesbed. The experimental evaluation over various channel conditions shows that GFA can significantly reduce the energy consumption by up to $6 \times$ compared to default Linux configuration. Moreover, the results show that GFA outperforms the static frame sizing method in terms of network goodput and preserves the end-to-end latency.

\section{REFERENCES}

[1] A. Showail, K. Jamshaid, and B. Shihada, "Buffer sizing in wireless networks: challenges, solutions, and opportunities," Communications Magazine, IEEE, Accepted, 2014.

[2] B. S. Kim, H. Hwang, and D. K. Sung, "Effect of frame aggregation on the throughput performance of IEEE 802.11n," in Wireless Communications and Networking Conference, 2008. WCNC 2008. IEEE, pp. 17401744, March 2008.

[3] K. Lee, S. Yun, and H. Kim, "Boosting video capacity of IEEE $802.11 \mathrm{n}$ through multiple receiver frame aggregation," in Vehicular Technology Conference, 2008. VTC Spring 2008. IEEE, pp. 2587-2591, May 2008.

[4] T. Selvam and S. Srikanth, "A frame aggregation scheduler for IEEE 802.11n," in Communications (NCC), 2010 National Conference on, pp. 1-5, Jan 2010.

[5] Y. Lin and V. Wong, "WSN01-1: Frame aggregation and optimal frame size adaptation for IEEE 802.11n WLANs," in Global Telecommunications Conference, 2006. GLOBECOM '06. IEEE, pp. 1-6, Nov 2006.

[6] P. Teymoori, A. Dadlani, K. Sohraby, and K. Kim, "An optimal packet aggregation scheme in delay-constrained IEEE 802.11n WLANs," in Wireless Communications, Networking and Mobile Computing (WiCOM), 2012 8th International Conference on, pp. 1-4, Sept 2012.

[7] M. Moh, T.-S. Moh, and K. Chan, "Error-sensitive adaptive frame aggregation in $802.11 \mathrm{n}$ WLAN," in Wired/Wireless Internet Communications, pp. 64-76, Springer, 2010.

[8] S. Keranidis, G. Kazdaridis, N. Makris, T. Korakis, I. Koutsopoulos, and L. Tassiulas, "Evolution of IEEE 802.11 compatible standards and impact on energy consumption," CoRR, vol. abs/1308.1963, 2013.

[9] S. Jeon and J. Lee, "Adaptive frame aggregation scheme for energy efficiency in WLAN," in Consumer Electronics (ICCE), 2011 IEEE International Conference on, pp. 463-464, Jan 2011.

[10] G. Zhihui, L. Anzhong, and L. Taoshen, "EEFA: Energy efciency frame aggregation scheduling algorithm for IEEE 802.11n wireless network," Communications, China, vol. 11, pp. 19-26, March 2014.

[11] A. Showail, K. Jamshaid, and B. Shihada, "An empirical evaluation of bufferbloat in IEEE 802.11n wireless networks," in Wireless Communications and Networking Conference (WCNC), 2014 IEEE, April 2014.

[12] T. Szigeti, C. Hattingh, F. Knox, A. To, and C. Varner, End-to-end QoS network design: Quality of Service in LANs, WANs, and VPNs. Cisco press Indianapolis, 2005.

[13] S. Keranidis, G. Kazdaridis, V. Passas, T. Korakis, I. Koutsopoulos, and L. Tassiulas, "Online energy consumption monitoring of wireless testbed infrastructure through the NITOS EMF framework," in Proceedings of the 8th ACM International Workshop on Wireless Network Testbeds, Experimental Evaluation and Characterization, WiNTECH '13, (New York, NY, USA), pp. 89-90, ACM, 2013.

[14] A. Showail, K. Jamshaid, and B. Shihada, "WQM: An aggregationaware queue management scheme for IEEE 802.11n based networks," in Proceedings of the 2014 ACM SIGCOMM Workshop on Capacity Sharing Workshop, CSWS '14, 2014. 